\title{
DESIGN OF SCANNING SYSTEM FOR MECHANOSCOPIC ANALYSIS
}

\author{
Milan Navrátil, Vojtěch Křesálek, Josef Kudělka, Tomáš Martínek \\ Faculty of applied informatics, Tomas Bata Univerzity in Zlin, Nad Stráněmi 4511, Zlin 76005, Czech Republic
}

\begin{abstract}
The work deals with hardware and software proposal of a system suitable for control and visualization of scanning process based on resistivity measurement. Three axis positioning system with conductive contact probe is utilized. The user application was created in MATLAB programming environment. Important part of the design represents the definition of requirements, choice of the appropriate tools, functions and structures needed for reliable realization. Proposed user application allows setting key parameters of the measurement, appropriate method of data acquisition as well as performing online 3D visualization of measured data. The functionality of the application is verified via measurement of a several selected samples. Results of these measurements are also presented.
\end{abstract}

Keyword: scanning; mechanoscopic analysis; MATLAB
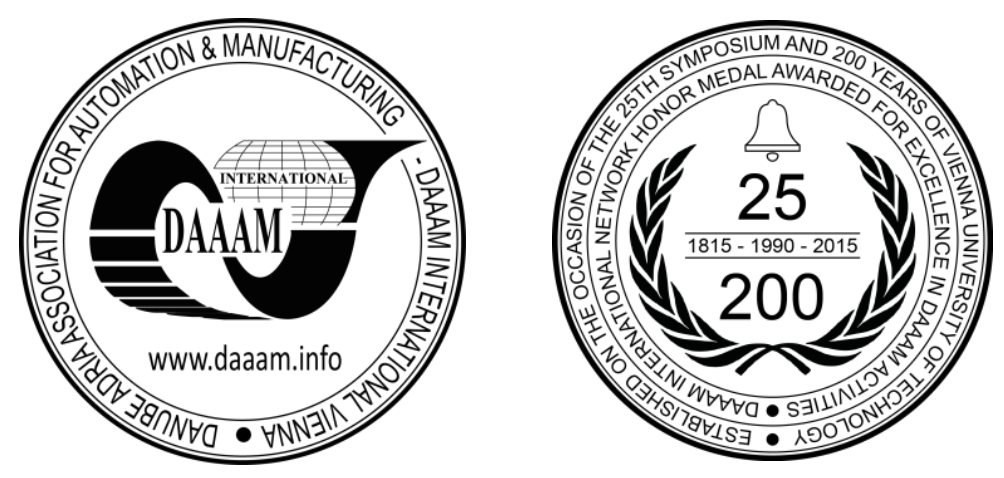

This Publication has to be referred as: Navratil, M[ilan]; Kresalek, V[ojtech]; Kudelka, J[osef] \& Martinek, T[omas] (2016). Design of Scanning System for Mechanoscopic Analysis, Proceedings of the 26th DAAAM International Symposium, pp.0256-0264, B. Katalinic (Ed.), Published by DAAAM International, ISBN 978-3-902734-07-5, ISSN 1726-9679, Vienna, Austria DOI:10.2507/26th.daaam.proceedings.035 


\section{Introduction}

In general, mechanoscopy is a field of criminology dealing with tool marks examination and identification. The first book concerning this area was written by Czech, a police chief sergeant Ladislav Havlicek called "Mechanoscopy: Traces and Signs of Craft Tools" in 1940. This handbook contained description of methods based on author's practical experience with the investigation of bank robberies [1]. A part of the mechanoscopy dealing with marks after shooting from a weapon originates from ballistics and is called criminological forensic ballistics [2]. If we focus on identifying of hand weapons commonly used in various types of crime in order to help in clarifying crimes, we need to examine marks which are left after shot, such as cartridge case, prime or shot itself. In case of cartridge cases, where some marks after firing pin and cartridge ejector can be observed. This observation can be accomplished, for example, by using of suitable methods such as optical or confocal microscopy. The result is in form of picture and some correlation methods can be applied for comparison of individual results [3], [4]. Our approach lies in scanning of the surface resulting in 3Dimage of the surface.

For imaging surfaces there are variety of different scanning methods and new variations are still growing. Their applicability is mainly based on used physical principle. This determines which method is or is not suitable for the specific application. When choosing the method we have to take into account the characteristics of the scanned material, method of display, precision requirements as well as required resolution [5]. Finally, the complexity of the implementation and the economic aspect are also important. Although numerous commercial scanning systems exist, some of the more interesting and challenging applications demand custom design. A common obstacle to custom design is the difficulty of creating custom data acquisition hardware and writing the complex steady software required to run the scanning system [6].

From historical point of view, the invention of scanning tunneling microscopy (STM) and later atomic force microscopy (AFM) permitted development of a new techniques, which are called scanning probe microscopy (SPM). This method covers a group of techniques that provide measurement of surface topography or surface properties with atomic resolution. SPM can be operated in different modes, allowing different properties to be measured at identical positions in a sample [7].

The invention of scanning tunneling microscopy is dated back to 1981, when Gerd Binnig and Heinrich Rohrer built the first scanning tunneling microscope [8]. This technique utilizes quantum-mechanical phenomenon of tunneling current. Electrons flow between surfaces of two materials which are not in contact. Binning and Rohrer subsequently used this method to resolve the structure of silicon at the atomic scale [9]. Key aspect of their experiment was a feedback loop that controlled the gap between the tip and measured sample. Usability of mentioned method is limited to conducting or semiconducting samples.

The STM measurement can be performed in two ways. The first one represents measurement setup where the tip is fixed and the sample is moved in predefined raster. In the second case, the sample is stationary and the tip is manipulated. In following text, we suppose using of the first case. During measurement, a coarse movement of the sample to a tip in the $\mathrm{Z}$ direction is done; this may be purely mechanical. After that, voltage is applied between the tip and the sample to flow the electric current. The gap between the tip and sample is decreasing due to very fine movement cause by a suitable positioning system until the current flow is measurable. Image acquisition (scanning) is gradually performed in two dimensions ( $\mathrm{x}-\mathrm{y}$ raster) using step change in position. The output of the measurement is represented by matrix Aij whose indices indicate the position of the point position and the relevant value means the measured signal. This signal can be of two kinds; depending on the measuring mode [10].

Constant height mode - the gap between tip and sample is adjusted and the tunneling current is measured. This mode allows very fast image scanning, because it is not necessary to move the sample in $\mathrm{Z}$ direction. On the other hand, it is less precise because of large tip distances from the sample surface which results in less electric current, often under measurable levels.

Constant current mode - a feedback is used to keep the constant current level. This mode is slower than the previous one but it allows to interrogate higher topographical changes in the surface profile. However, it depends on the relationship between the applied voltage and change of piezo-element extent. This dependence can be removed by the outside position measurement, for example using laser. Another disadvantage lies in risk of damage the surface when the tip moves over the area with some significantly different electrical properties (oxidized spots).

To achieve high resolution, it is required to have a tip as sharp as possible, in other words, tip with small curvature radius, preferably only one atom at the tip apex of the cone. It is due to preventing of superposition of individual interactions. However, it is difficult to produce such tip, but due to mentioned dependency it is not necessary. The tip with macroscopic curvature is enough, because there is always one atom outstanding through it then almost all current flows. Resolution also depends on the scanning step size, which is the limiting parameter mainly in micrometer dimensions. The step size depends inversely on the number of measuring points which affects measurement time of one complete image. Choosing the suitable step is a compromise between maximum resolution and the duration of interaction, which is important especially in the study of dynamic phenomena.

In case of mechanoscopic analysis, the size of traces is many times greater than STM or AMF methods can analyze. For AFM systems, the maximal size of scanned area rarely exceeds 100 micrometers. Another limitation is maximal height of the sample which is often about 1 centimeter. From reasons mentioned above, there is a need to modify or design other scanning systems allowing to measure larger samples. 


\section{Used instruments}

\subsection{Mercury $M 1101 D G$}

Mercury M-110 is a servo motor with a very high motoric resolution which enables linear motion in the range of $5 \mathrm{~mm}$. In contrast to conventional engines it allows precise adjustment axis position. The manufacturer guarantees a resolution of 0.5 micrometers but the minimum possible displacement is several times smaller [11], [12]. Maximum speed is $1 \mathrm{~mm} / \mathrm{s}$. The advantage is the possibility of a combination of several M-110 so that the defined movement in several axes. Stepper drivers are needed to control the displacement in respective axes.

\subsection{Mercury C-862}

It is a networkable single-axis DC-motor controller which uses the feedback quadratic encoder signal to determine the position. Depending on the decomposition encoder scale we can achieve precision motion down to 0.05 micrometers. When using multiple Mercury M110 together with C862 there is a need to connect each step drivers into the network via RS-232. The whole system is also connected via RS-232 interface and controlled with the computer. Each individual step driver is individually addressed. Before the command broadcasting there is therefore necessary to select which driver the command is specified for.

\subsection{Hewlett Packard 34401A}

This digital multimeter has a maximum resolution of $61 \frac{1}{2}$ digit. The relative measurement uncertainty is for $\mathrm{AC}$ values of $0.06 \%$ and $0.0015 \%$ for DC values. The device allows measurement of up to $1 \mathrm{kV}$. Measurement bandwidth is from $3 \mathrm{~Hz}$ to $300 \mathrm{kHz}$. Measurement speed reaches to 1000 measurements per second depending on the number of digits in the measurement. This laboratory multimeter was used for resistance measurement during scanning procedure.

\subsection{Scanning probe}

Indispensable part of scanning method is a scanning probe (a tip). Point radius and size are key properties which limit the maximum possible resolution that can be achieved. During measurement, it is suitable to attach the tip in a way to have possibility of free movement in Z-axis. This is because of the physical contact between the sample and the tip. It does not always lead to the evaluation of the contact (for example, impurity in the sample prevents current flow). The sample is then still moving towards the tip until the detection of changes in resistance. This can cause either damage of the tip or partial damage of the sample. However, it must be ensured that the tip is always returned to the same position, otherwise the result was considerably distorted. To prevent mentioned negative aspects, the tip was placed in a tightly clamped housing, which enables free movement of the tip in the vertical direction. In our case, the sample was fixed on movable stage and the probe (tip together with housing), see Fig. 1a. Used probe has a slim design, small flat tip, with size of 35 micrometers. This size is important for determination of the minimum distances between grid points, which should be at least twice this size.

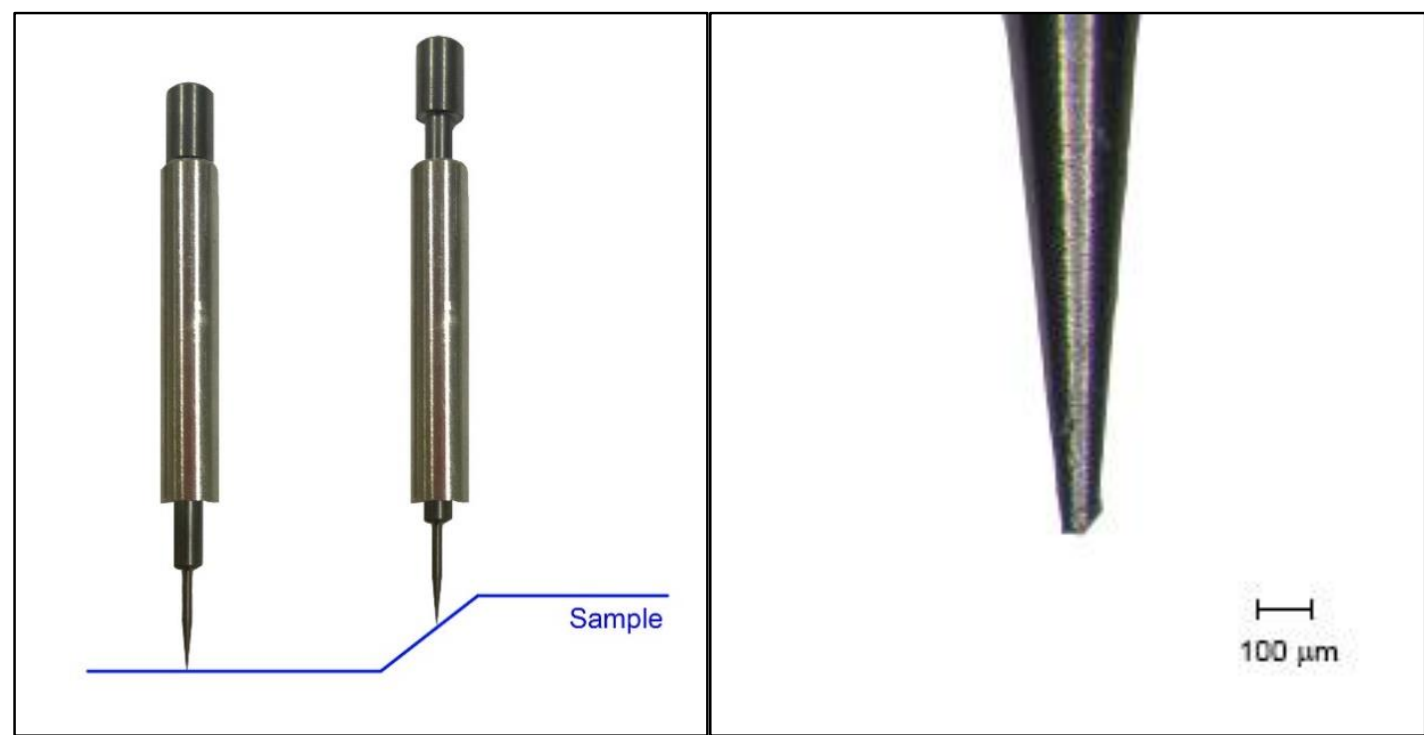

Fig. 1. (a) Free movement of the tip in vertical direction to prevent damage; (b) Stereo-microscopic picture of the tip 


\section{Measurement setup}

Servomotors together with their controllers are connected to personal computer via serial port RS-232. Multimeter is connected via GPIB interface. Remote control of all used instruments is performed using SCPI commands according user or operating manuals.

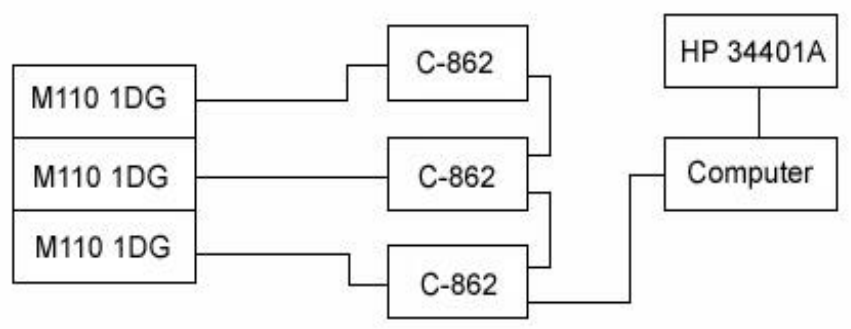

Fig. 2. Scheme of connection of the used instruments (RS232 or GPIB interface is utilized)

For scanning process, the contact method using resistance measurement was used. The multimeter measures the resistance of the electric circuit as it is shown below in Fig. 3a and flowchart feedback system in Fig. 3b. Displayed switch is represented by a stationary scanning sharp tip together with a conductive sample which is examined. The sample is connected to the plate which is fixed to the stage. Both two parts, sample and plate, must be conducting. Movements of the sample is performed via servo motors equipped with the controllers. Maximal travel range in individual axis is $5 \mathrm{~mm}$, minimal incremental motion is $50 \mathrm{~nm}$. The resistor R1 is connected in parallel to the switch. When the tip with the sample is in contacts then the switch is on. As a result, the resistance in the circuit is decreased. The resistor values were chosen as follows: $\mathrm{R} 1=1 \mathrm{M} \Omega, \mathrm{R} 2=100 \Omega$.
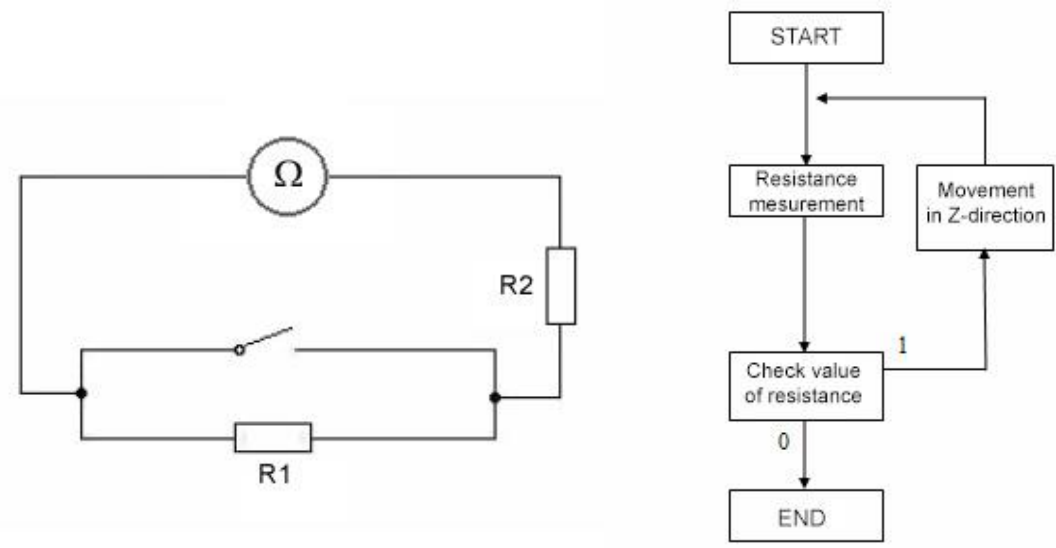

Fig. 3. Used feedback system (a) electric scheme; (b) flowchart

Whole measurement setup can be seen in Fig. 4 where the tightly fixed conductive contact probe is set to measure cartridge case after shooting. The trace after firing pin is examined. Detailed results from this measurement is presented below.

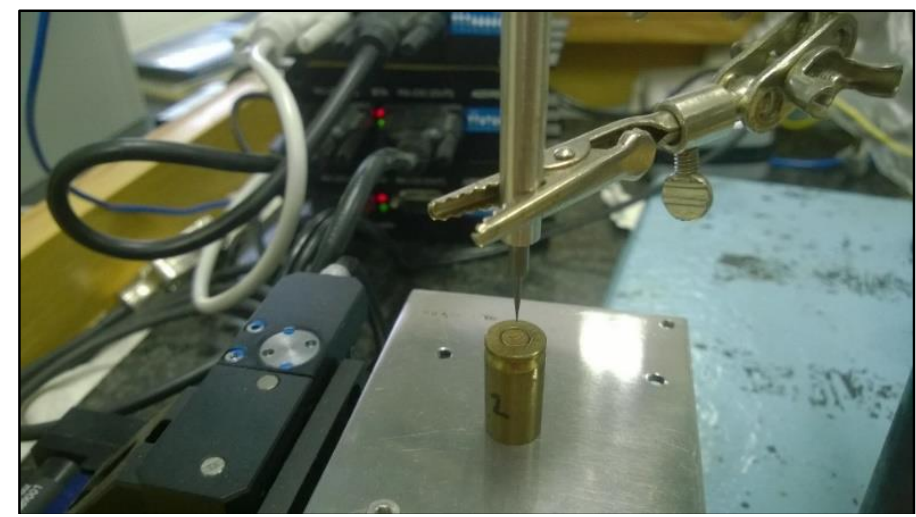

Fig. 4. Detail view on scanning system - conducting contact probe is stationary, stage with the sample is moveable in three axes 


\section{Software design}

Despite the motor producer, Physik Instrumente Company, provides simple software called MMCRun (including macro manager) to communicate with controller and control the motor, to implement other measuring devices cooperating with the motors is not feasible. There was a need to develop own user application which allows creating movement conditions on the base of measured resistance, parameter settings and online showing of measured data in form of 3D-visualization with graphical output.

The application was created in MATLAB environment using graphical user interface (GUI). Communication between user application and connected instruments is intermediated via Control instrument toolbox. Servomotor's manufacturer provided set of remote commands (SCPI) which are described in individual manuals. The principle of the program can be described as follows. There are three interrelated cyclic functions. The first one provides the movement along the rows ( $\mathrm{X}$ axis), the second in columns ( $\mathrm{Y}$-axis) and the third ensures contact between the sample and the scanning tip (movement in the $\mathrm{Z}$ direction). The sample is very finely approaching towards to the tip, when contact is detected, the movement immediately stops and positions of all three axes are obtained. They are stored in the format of $\{\mathrm{X}$ value, $\mathrm{Y}$ value, $\mathrm{Z}$ value $\}$ for later processing. After saving the current data, the sample is quickly moved away from the tip to default position in vertical direction. After that the sample is moved to the next measuring point (next column). After whole line is completed, the sample is moved to the beginning of the next line and so on. Movements of the stage in X-Y plane is graphically expressed in Fig. 5. After measuring all the lines (all the specified grid points), the sample is moved away from the tip and the measurement is finished.

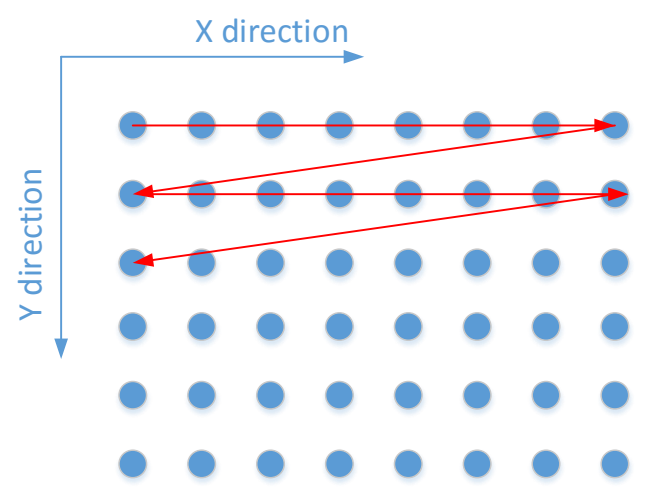

Fig. 5. Step by step measuring in X-Y raster

a

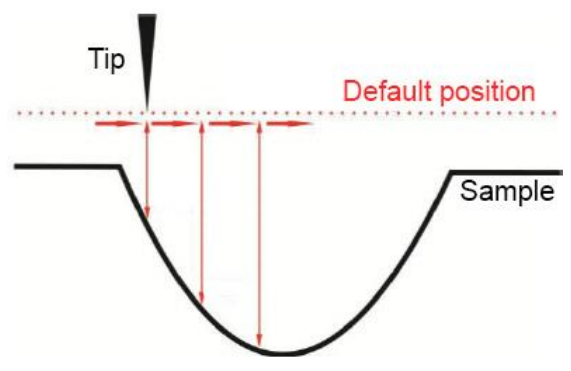

b

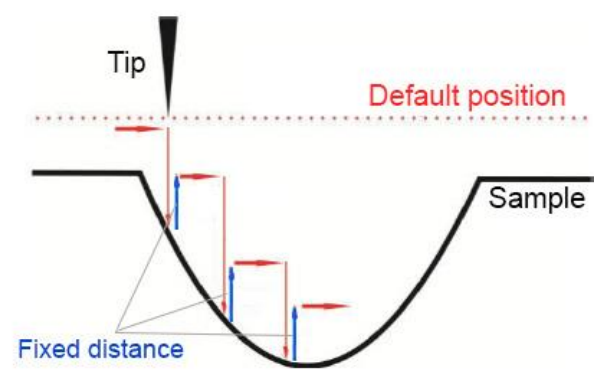

Fig. 6. Vertical return position of the tip: (a) default; (b) fixed distance

Disadvantage of the mentioned principle lies in very long measurement time especially when choosing higher resolution. To decrease measurement time, some improvements were performed. Instead of movement from contact position towards to default position in Z-axis, the sample is moved from the tip by defined fixed distance, see Fig. 6. This value should be set very sensitively due to possible collision of the sample with the tip. The surface topology of interrogated sample also influences mentioned value. Big advantage of this system is represented by adjustable working distance between the tip and the sample. In this case we are not limited by a maximal distance. According set parameters the estimated total time is calculated and shown.

The key parameters which are set in user application are:

- Number of measuring points within the $\mathrm{X}$-axis [-]

- Number of measuring points within the Y-axis [-]

- Distance between two neighboring measuring points in $\mathrm{X}$-axis $[\mu \mathrm{m}]$

- Distance between two neighboring measuring points in $\mathrm{Y}$-axis $[\mu \mathrm{m}]$

- Approaching step $[\mu \mathrm{m}]$

- Fixed distance $[\mu \mathrm{m}]$

- Approaching velocity $[\mu \mathrm{m} / \mathrm{s}]$ 


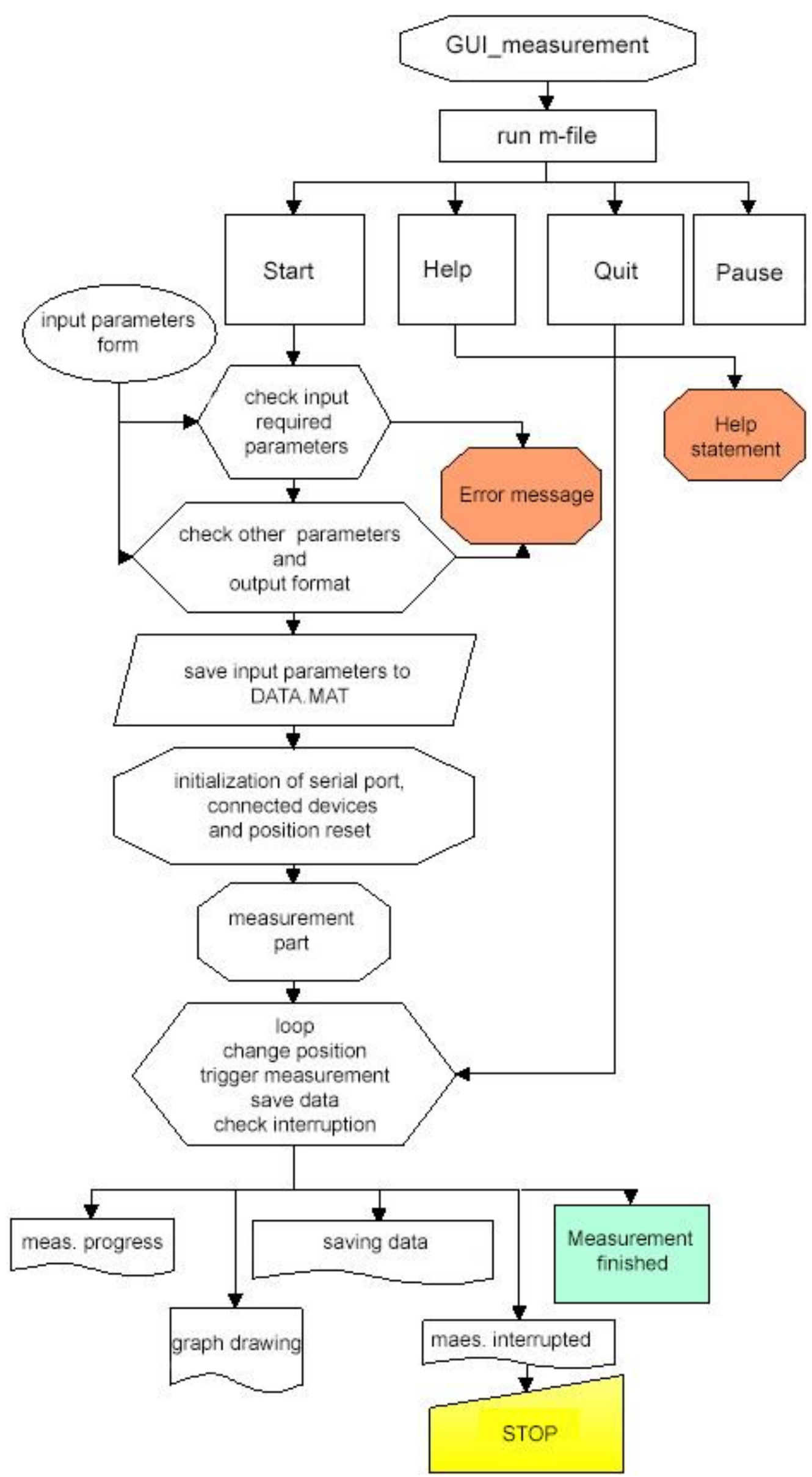

Fig. 7. Completed flowchart of the user application implemented in MATLAB 


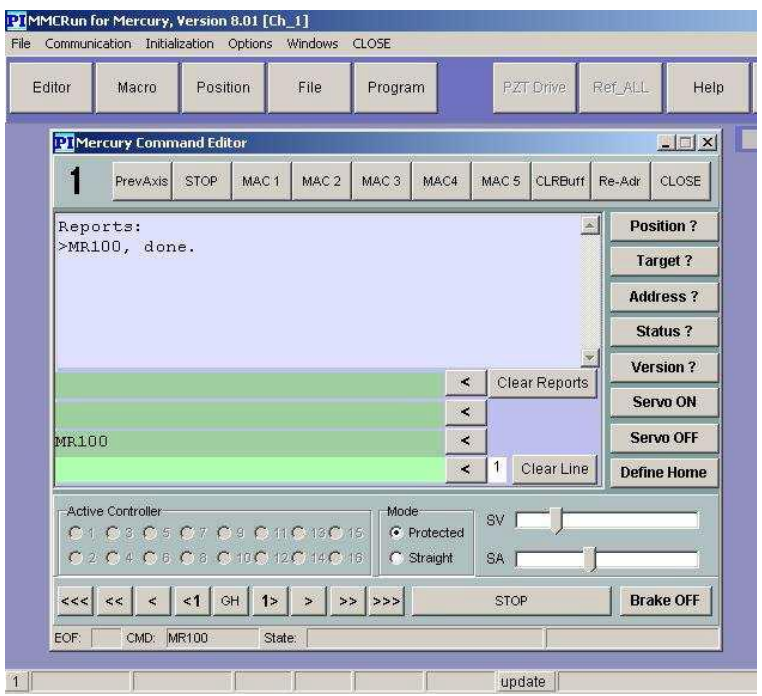

b

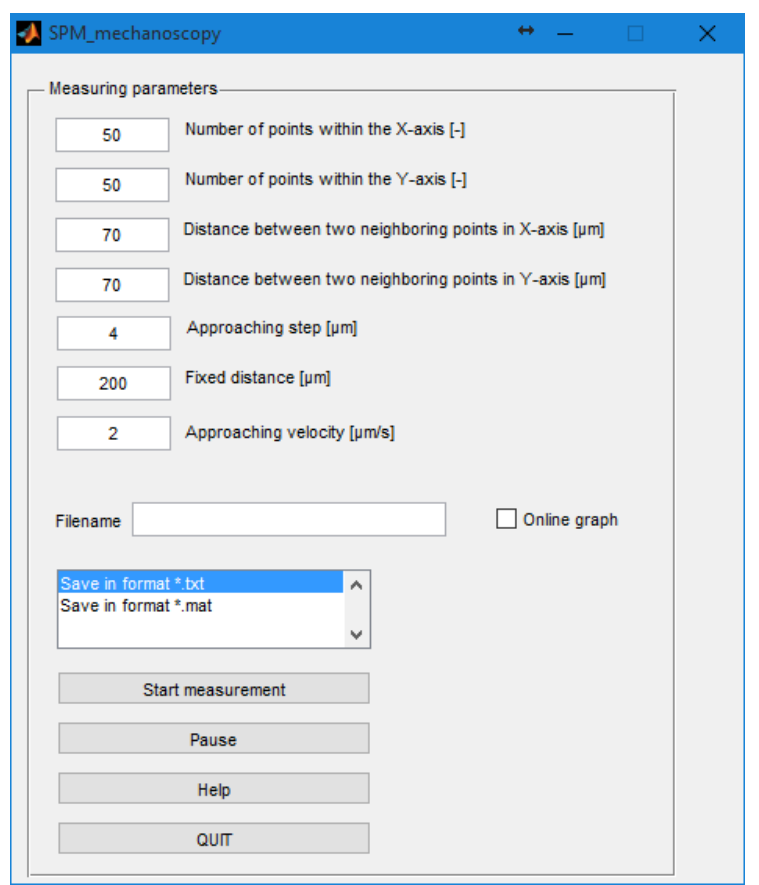

Fig. 8. Screenshot of the main window (a) MMCRun software from PI Company; (b) created user application SPM_mechanoscopy

\section{Test measurement}

The area around number zero on the tail of $1 \mathrm{CZK}$ coin was chosen for 3D-visualisation, it is highlighted and shown in Fig. 9. Number of measuring points within the $\mathrm{X}$-axis and $\mathrm{Y}$ axis was 36, distances between two neighboring measuring points in $\mathrm{X}$-axis and $\mathrm{Y}$-axis was $60 \mu \mathrm{m}$, approaching step was $2 \mu \mathrm{m}$. Total measuring time was approximately 20 hours in case of default return position. After optimization with fixed distance value of $50 \mu \mathrm{m}$ the measurement time decreased to 12 hours.

a

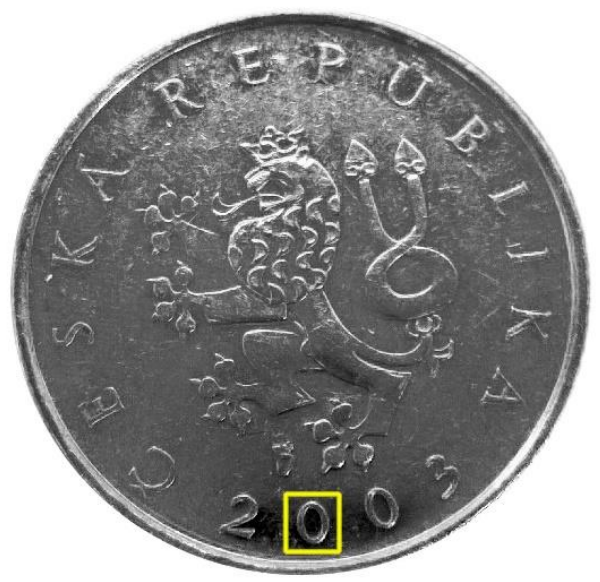

b

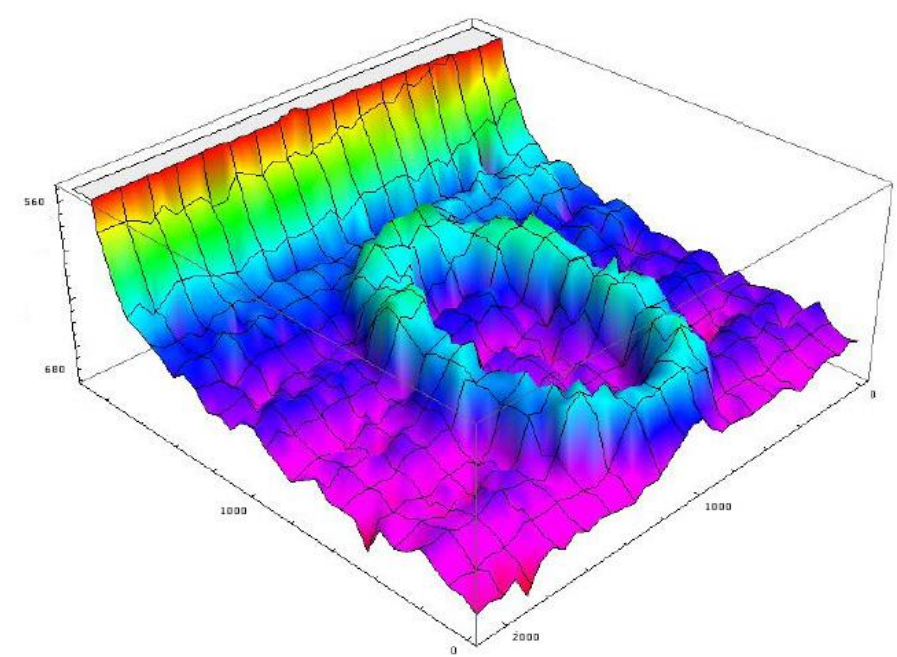

Fig. 9. (a) 1 CZK coin with highlighted scanned area; (b) 3D-visualization of the scanned area (number " 0 " on the tail of coin)

The area around number zero on the tail of $1 \mathrm{CZK}$ coin was chosen for 3D-visualisation, it is highlighted and shown in Fig. 9. Number of measuring points within the $\mathrm{X}$-axis and $\mathrm{Y}$ axis was 36, distances between two neighboring measuring points in $\mathrm{X}$-axis and $\mathrm{Y}$-axis was $60 \mu \mathrm{m}$, approaching step was $2 \mu \mathrm{m}$. Total measuring time was approximately 20 hours in case of default return position. After optimization with fixed distance value of $50 \mu \mathrm{m}$ the measurement time decreased to 12 hours. 

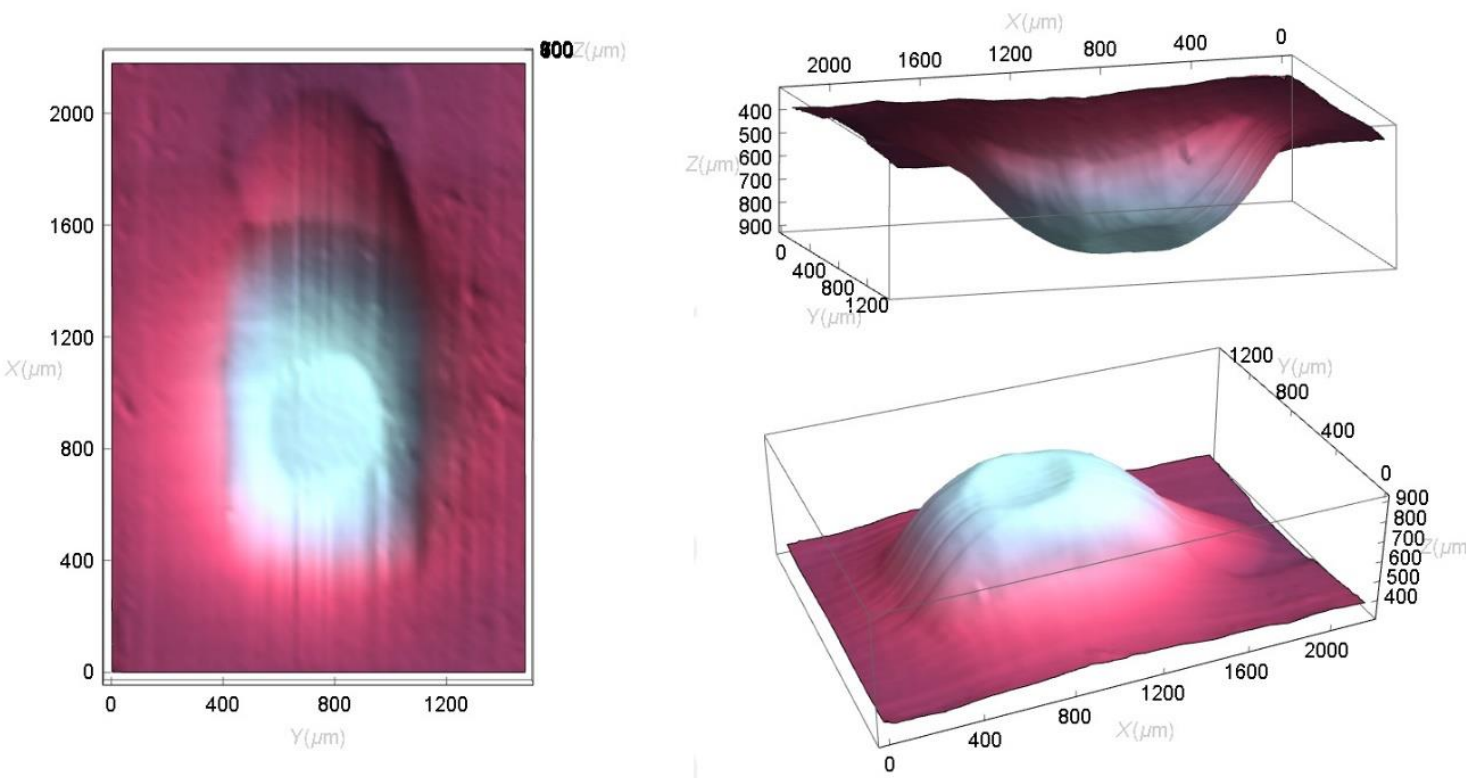

Fig. 10. 3D visualization of the cartridge case after shooting in different point of view

\section{Conclusion}

Although numerous commercial scanning systems exist, some of the more interesting and challenging applications demand custom design. A common obstacle to custom design is the difficulty of creating custom data acquisition hardware and writing the complex steady software required to run the scanning system.

Our custom scanning system equipped with 3-axis compact micro-translation stage, conducting contact probe as well as user application "SPM_mechanoscopy" in MATLAB programming environment were designed and described. The necessary feedback of the system was based on contact resistance change measurement using multimeter HP 34401A. User application allows setting key parameters for scanning measurement, showing online 3Dvisualization and estimation of total measuring time. The test measurement was carried out on the Czech coin (1 CZK). This measurement was accomplished to verify using of contact method and optimizing scanning process and also user program to reliably and effectively work. Another test measurement was performed. It concerned of measuring trace after firing pin of the specific gun after shooting which is important aspect in forensic ballistics area which is an important part of mechanoscopy. In contrast to optical methods, this scanning system allows us getting 3D structure of the scanned object, which can be further mathematically modelled and consequently compared to other objects. Obtained results are sufficient for coarse description of surface topography but there is a crucial relation between resolution and time measurement. The resolution in micrometer ranges is affected by macroscopic shape of the tip due to its close proximity to the surface sample.

Advantages of used system are represented by high accuracy and smoothness of movement, simple operation, possibility of creation of mathematical model followed by comparison of samples (shape, depth) and their 3D visualization. Moreover, in case of mechanoscopy, the size of traces is many times greater than STM or AMF methods can analyze. For AFM systems, the maximal size of scanned area rarely exceeds 100 micrometers. Another limitation is maximal height of the sample itself which is often about 1 centimeter. Our SPM method is very suitable for mechanoscopic analysis due to possibility of working distance adjusting between the tip and the sample so that we can measure some small parts of large samples. This distance can be set in decimeter units. On the other hand, time demands of this measurement is still very high despite some optimizing techniques. There is another limitation in necessity of use only conducting samples together with contact conducting probe. Disadvantage also lies in necessity of good contact between the scanning tip and the sample surface. Slight dirt on sample surface can lead to incorrect evaluation of resistivity condition. To improve this, there is a need to very gently clean the surrounding of scanned area but in a way the sample is not corrupted or damaged, especially in case of soft samples.

In future, we are planning data processing improvement such as equalization of incident plane or creating custom cut planes. However, the biggest impact on quality of the scanned image has chosen raster together with geometry of the tip and the material which is made of. The sharpest tip we have, the smallest details we can resolve. From the hardware point of view, the replacement of the used feedback system can surely lead enlargement of measurement possibilities.

\section{Acknowledgements}

This work was supported by the Ministry of Education, Youth and Sports of the Czech Republic within the National Sustainability Programme project No. LO1303 (MSMT-7778/2014). 


\section{References}

[1] C. A. Brebbia. Structural studies, repairs and maintenance of heritage architecture XIII. WIT Press, 2013,594 pages. ISBN 978-184-5647-308.

[2] V. Dimaio. Gunshot wounds: practical aspects of firearms, ballistics, and forensic techniques. CRC press, 1998.

[3] C. L. Smith. Fireball: a forensic ballistics imaging system. Proceedings IEEE 31st Annual 1997 International Carnahan Conference on Security Technology. IEEE, 1997, : 64-70. DOI: 10.1109/CCST.1997.626240. ISBN 07803-3913-4.

[4] M. S. Bonfanti, R. I. Ghauharali. Visualisation by confocal microscopy of traces on bullets and cartridge cases. Science. 2000, 40(4): 241-256. DOI: 10.1016/S1355-0306(00)71994-0. ISSN 13550306.

[5] V. Tsukruk, S. Singamaneni. Scanning probe microscopy of soft matter: fundamentals and practices. Weinheim: Wiley-VCH, c2012. ISBN 978-3-527-32743-0.

[6] T. A. Pologruto, B. L. Sabatini, K. Svoboda. (2003). ScanImage: flexible software for operating laser scanning microscopes. Biomed. Eng. Online, 2(1), 13.

[7] R. Berger, H.J. Butt, M.B. Retschke, A. L. Weber. Electrical Modes in Scanning Probe Microscopy. Macromolecular Rapid Communications. 2009, 30(14): 1167-1178. DOI: 10.1002/marc.200900220. ISSN 10221336.

[8] G. Binnig, H. Rohrer, Ch. Gerber, E. Weibel. "Surface studies by scanning tunneling microscopy," Phys. Rev. Lett., vol. 49, no. 1, pp. 57-61, 1982.

[9] G. Binnig, H. Rohrer, Ch. Gerber, E. Weibel. "7 77 reconstruction on si(111) resolved in real space," Phys. Rev. Lett., vol. 50, no. 2, pp. 120-123, 1983.

[10] P. Hawkes, J. Spence. Science of microscopy. New York: Springer, c2007, 2 v. (xviii, 1265, I26 p.). ISBN 03-8725296-7.

[11] MS 74E User Manual. Physik Instrumente. Mercury C-862 Networkable Single-Axis DC-Motor Controller. Release 8.41. Karlsruhe, 2004

[12] Physik Instrumente, Piezo nano positioning, Product Overview: Piezo Systems, Precision Motion Control, Micro \& Nanopositioning [online]. 2013 [cit. 2015-09-05]. Available at: http://www.physikinstrumente.com/en/index.php

Service Guide. Agilent Technologies. Agilent 34401A 61ํ2 Digit Multimeter [online]. Seventh edition. Santa Clara, 2007 [cit. 2015-09-15]. Available at: WWW:http://www.home.agilent.com/agilent/product.jspx?cc=CZ\&lc=end\&nid=536902435.536880933\&pageMode=PLublishing Inc., New York, 1999, pp. 281-304.

[13] G. Gramse et al. Calibrated complex impedance and permittivity measurements with scanning microwave microscopy. Nanotechnology, 2014, 25.14: 145703.

[14] I. Baldea. Concurrent conductance and transition voltage spectroscopy study of scanning tunneling microscopy vacuum junctions. Does it unravel new physics?. RSC Advances, 2014, 4.63: 33257-33261. 